\title{
Balanced Lines, Halving Triangles, and the Generalized Lower Bound Theorem
}

\author{
Micha Sharir \\ Emo Welzl
}

\begin{abstract}
A recent result by Pach and Pinchasi on so-called balanced lines of a finite two-colored point set in the plane is related to other facts on halving triangles in 3-space and to a special case of the Generalized Lower Bound Theorem for convex polytopes.
\end{abstract}

\section{Introduction}

The following three facts are related to each other.

Fact $\mathbf{A}$ Let $R$ and $B$ be two disjoint finite planar sets, so that $|R \cup B|=2 n$ is even and $R \cup B$ is in general position (i.e., no three points are collinear). Points in $R$ and $B$ are referred to as 'red' and 'blue,' respectively. A line $\ell$ is balanced (w.r.t. $(R, B))$ if $\ell$ passes through a red point and a blue point, and on both sides of $\ell$, the number of red points minus the number of blue points is the same.

The number of balanced lines is at least $\min \{|R|,|B|\}$.

If $R$ and $B$ can be separated by a line (but also in other configurations), this number is attained.

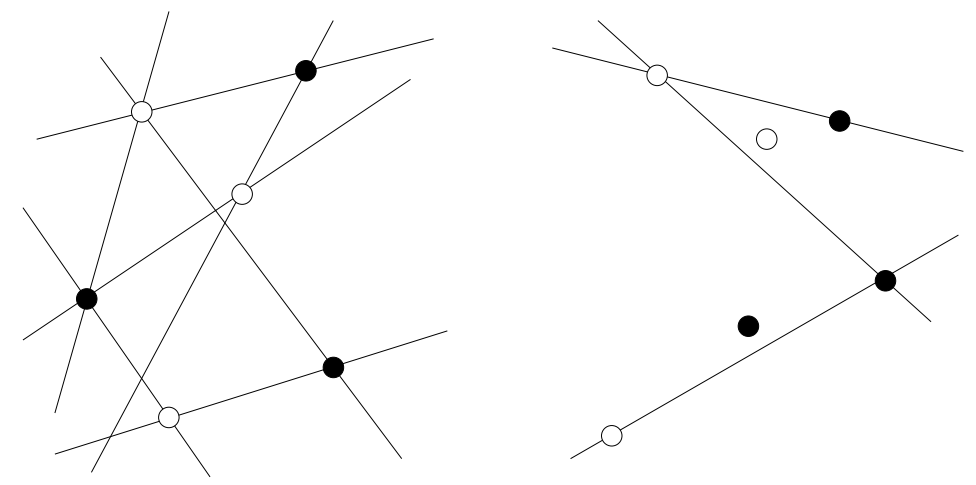

Figure 1: Balanced Lines.

Fact B $n \in \mathbf{N}$. Let $Q$ be a set of $2 n+1$ points in 3 -space in general position (i.e., no four points are coplanar). A halving triangle of $Q$ is a triangle spanned by three points in $Q$ such that the plane containing the three points equipartitions the remaining points of $Q$.

The number of halving triangles is at least $n^{2}$.

If $Q$ is in convex position (but also in other configurations), this number is attained.

Fact $\mathbf{C} \quad d \in \mathbf{N}$, even ${ }^{1}$. Let $\mathcal{P}$ be a convex polytope which is the bounded intersection of $d+4$ halfspaces

\footnotetext{
${ }^{1}$ For reference to previous and forthcoming facts: $d+4=m=2 n$.
} 
in general position in $d$-space, i.e., no $d+1$ bounding hyperplanes meet in a common point. (Therefore, either $\mathcal{P}$ is empty, or it is a simple convex $d$-polytope with at most $d+4$ facets. All vertices are incident to $d$ edges. Our set-up is chosen in this way, in order to have a clean relation to the other statements.) Let its edges be oriented according to a generic linear function (edges are directed from smaller to larger value; 'generic' means that the function evaluates to distinct values at the vertices of $\mathcal{P}$ ).

The number of vertices with $d / 2-1$ outgoing edges is at most the number of vertices with $d / 2$ outgoing edges.

If $\mathcal{P}$ is empty (but also for other polytopes), this is tight.

(In fact, for any $d$ (whether odd or even), and for all $1 \leq j \leq\lceil d / 2\rceil$, the number of vertices with $j-1$ outgoing edges is at most the number of vertices with $j$ outgoing edges. And for $d$ odd, and $j=\lceil d / 2\rceil$, these numbers are even equal. But that will not be relevant in our context.)

(A) has been proved by J.Pach and R. Pinchasi [7], answering a question of G. Baloglou's. (The statement in [7] is restricted to the case $|R|=|B|=n$. Then a balanced line must have the same number of red and blue points on each side, and there are at least $n$ such balanced lines. But see Remark 2.1 below.) (C) is a very special case of the Generalized Lower Bound Theorem (GLBT) for simple polytopes, whichin turn - is part of the necessity part of the $g$-Theorem proved by R. P. Stanley [8] (thereby answering a conjecture by P. McMullen, who later provided also an alternative proof [6]); cf. also [10]. It was recently shown that $(B)$ and $(C)$ can be derived from each other [9]. In Section 2 we present a simple proof of the equivalence $(\mathrm{A} \Leftrightarrow \mathrm{B})$. That is, $(\mathrm{A})-(\mathrm{C})$ are equivalent to each other. ${ }^{2}$ In Section 3 , we give an alternative proof of the equivalence $(A \Leftrightarrow C)$. Clearly, that is already implied by $(A \Leftrightarrow B \Leftrightarrow C)$, but we include here an argument for this specific setting for the sake of completeness.

On one hand, this means that the result of [7] admits a proof that is considerably simpler than their original proof, via the GLBT. On the other hand, Pach and Pinchasi's proof has merits of its own, because (i) no purely combinatorial proof of the GLBT (such as that in [7]) has been previously known (not even for the special case $(\mathrm{C})$ equivalent to the balanced line problem), and (ii) that proof is based on allowable sequences in the dual, and thus (A) applies also for oriented matroids.

\section{Balanced Lines and Halving Triangles}

We first transform the balanced lines problem (A) to yet another problem (D) involving halving triangles in three dimensions, which appears to be new.

Assume that the points of $R \cup B$ (as in (A)) lie in the plane $z=1$. Map these points onto the unit sphere centered at the origin $O$ by: $R \ni r \mapsto r^{*}:=r /\|r\|$, and $B \ni b \mapsto b^{*}:=-b /\|b\|$. Let $S_{0}$ denote the resulting set of projected points, and put $S=S_{0} \cup\{O\}$. By a small perturbation of $R \cup B$ that does not change the combinatorial type of this set, we may assume that $S$ is in general position.

Observe the following properties, whose proofs are straightforward:

(i) The $x y$-plane, $\pi_{0}: z=0$, separates $S_{0}$ into sets of cardinalities $|R|$ and $|B|$.

(ii) For $r \in R$ and $b \in B$, the line passing through $r$ and $b$ is a balanced line iff the triangle $O r^{*} b^{*}$ is a halving triangle of $S$. In particular, this establishes a correspondence between the balanced lines in $R \cup B$ and those halving triangles of $S$ that are incident to $O$ and are crossed by $\pi_{0}$ (i.e., $\pi_{0}$ intersects their relative interior).

(iii) The point $O$ is an extreme point of $S$ if and only if $R$ and $B$ are separated by a line.

Moreover, we can apply a reverse transformation as follows. Let $Q$ be any set of $2 n+1$ points in 3 -space in general position. Let $q_{0} \in Q$ be a fixed point, and let $\pi_{0}$ be a plane of $Q$ that passes through $q_{0}$ and

\footnotetext{
${ }^{2}$ Of course, true statements are always equivalent; we mean that these facts can be derived from each other in a fashion that is significantly simpler compared to the proofs of the individual statements.
} 
through no other point of $Q$. Let $\pi$ be a plane parallel to $\pi_{0}$. Map each point $q \in Q \backslash\left\{q_{0}\right\}$ to the point of intersection of $\pi$ with the line that passes through $q$ and $q_{0}$. Denote by $R$ (resp. $B$ ) the subset of points on $\pi$ that are images of points of $Q$ that lie in the side of $\pi_{0}$ that contains (resp. does not contain) $\pi$.

(iv) A triangle $q_{0} q_{1} q_{2}$, for $q_{1}, q_{2} \in Q$, is a halving triangle crossed by $\pi_{0}$ if and only if the line that passes through the images of $q_{1}$ and $q_{2}$ is a balanced line w.r.t. $(R, B)$.

These properties imply the equivalence $(\mathrm{A} \Leftrightarrow \mathrm{D})$ of the result of Pach and Pinchasi and the following assertion (D).

Fact $\mathbf{D} n \in \mathbf{N}$. Let $Q$ be a set of $2 n+1$ points in 3-space in general position. Let $q_{0} \in Q$ be a fixed point, and let $\pi_{0}$ be a plane of $Q$ that passes through $q_{0}$ and through no other point of $Q$, and separates $Q \backslash\left\{q_{0}\right\}$ into two sets of cardinalities $k$ and $2 n-k$.

There are at least $\min \{k, 2 n-k\}$ halving triangles of $Q$

that are incident to $q_{0}$ and are crossed by $\pi_{0}$.

If $q_{0}$ is an extreme point of $Q$ (but also in other situations), this number is attained.

Let us first show that, indeed, for $q_{0}$ extreme, the number of halving triangles of $Q$ that are incident to $q_{0}$ and are crossed by $\pi_{0}$ equals $\min \{k, 2 n-k\}$. Project $Q_{0}=Q \backslash\left\{q_{0}\right\}$ centrally from $q_{0}$ onto a plane parallel to a supporting plane of $Q$ at $q_{0}$; denote the projected set by $Q_{0}^{*}$. The plane $\pi_{0}$ projects to a line $\lambda$ that separates $Q_{0}^{*}$ into sets of cardinalities $k$ and $2 n-k$. It is then easy to check that, for points $q_{1}, q_{2} \in Q_{0}$, the triangle $q_{0} q_{1} q_{2}$ is a halving triangle of $Q$ crossed by $\pi_{0}$ if and only if the segment $q_{1}^{*} q_{2}^{*}$, connecting the images $q_{1}^{*}, q_{2}^{*}$ of $q_{1}, q_{2}$, is a halving edge ${ }^{3}$ of $Q_{0}^{*}$ that is crossed by the line $\lambda$. By Lovász' Lemma $[3,5]$, the number of such edges is exactly $\min \{k, 2 n-k\}$.

We proceed to a proof of implication ( $D \Rightarrow B$ ). Suppose (D) holds. Consider a set $Q$ of $2 n+1$ points. Let $\pi_{q}$, for $q \in Q$, be pairwise parallel planes such that $\pi_{q} \cap Q=\{q\}$ for each $q \in Q$. Every halving triangle $\Delta$ of $Q$ is crossed by exactly one of these planes which is also incident to a vertex of $\Delta$ (a plane crosses a triangle if it contains one of the three vertices, and separates the other two). Hence, there are at least

$$
\sum_{i=1}^{2 n+1} \min \{i-1,2 n+1-i\}=n^{2}
$$

halving triangles, which implies (B). (By the preceding argument, equality is attained when $Q$ is in convex position.)

Finally, let us provide the proof of implication $(B \Rightarrow D)$. Suppose that assertion (D) is false. Thus there exist a set $Q$ of $2 n+1$ points, a parameter $0 \leq k \leq 2 n$, a point $q_{0} \in Q$ and a plane $\pi_{0}$ passing through $q_{0}$ and partitioning $Q \backslash\left\{q_{0}\right\}$ into two sets of cardinalities $k$ and $2 n-k$, such that the number $c$ of halving triangles of $Q$ incident to $q_{0}$ and crossed by $\pi_{0}$ is strictly smaller than $\min \{k, 2 n-k\}$. First, we project $Q_{0}=Q \backslash\left\{q_{0}\right\}$ from $q_{0}$ onto a sphere centered at $q_{0}$; let $Q_{0}^{\prime}$ denote the resulting set of projected points, and $Q^{\prime}=Q_{0}^{\prime} \cup\left\{q_{0}\right\}$. In this way, the collection of halving triangles incident to $q_{0}$ did not change, nor did the number of points on either side of $\pi_{0}$. Therefore $Q^{\prime}, q_{0}$ and $\pi_{0}$ still provide a configuration contradicting (D). Now let $\pi_{q}$, for $q \in Q_{0}^{\prime}$, be planes parallel to $\pi_{0}$ with $\pi_{q} \ni q$ for each $q$. If necessary, rotate $\pi_{0}$ slightly about $q_{0}$ so that $\pi_{q} \cap Q^{\prime}=\{q\}$ for each $q \in Q_{0}^{\prime}$. As in the previous argument, every halving triangle of $Q^{\prime}$ is crossed by exactly one of the planes in $\left\{\pi_{0}\right\} \cup\left\{\pi_{q} \mid q \in Q_{0}^{\prime}\right\}$ (which is also incident to a vertex of the triangle). Since all points apart from $q_{0}$ are extreme in $Q^{\prime}$, the number of halving triangles of $Q^{\prime}$ is exactly

$$
\underbrace{c-\min \{k, 2 n-k\}}_{<0}+\underbrace{\sum_{i=1}^{2 n+1} \min \{i-1,2 n+1-i\}}_{=n^{2}}<n^{2} .
$$

The equivalence $(\mathrm{B} \Leftrightarrow \mathrm{D})$, and thus $(\mathrm{B} \Leftrightarrow \mathrm{A})$ is established.

\footnotetext{
${ }^{3}$ An edge whose containing line equipartitions $Q_{0}^{*} \backslash\left\{q_{1}^{*}, q_{2}^{*}\right\}$.
} 
Remark 2.1. Consider $Q_{0}^{\prime} \cup\left\{q_{0}\right\}$ as in the argument just given. Let $\pi^{\prime}$ be another plane through $q_{0}$ that partitions $Q_{0}^{\prime}$ into sets of cardinalities $k^{\prime}$ and $2 n-k^{\prime}$, and let $c^{\prime}$ be the number of halving triangles incident to $q_{0}$ and crossed by $\pi^{\prime}$ (this is also the number of such halving triangles in the original $Q$ ). Since the left-hand side of (1) is equal to the number of halving triangles of $Q_{0}^{\prime} \cup\left\{q_{0}\right\}$, it follows that

$$
c-\min \{k, 2 n-k\}=c^{\prime}-\min \left\{k^{\prime}, 2 n-k^{\prime}\right\} .
$$

Hence, if there were a configuration contradicting (D), then there would also be one with a plane $\pi_{0}$ that equipartitions $Q \backslash\left\{q_{0}\right\}$, and, thus, if there were a configuration contradicting (A), then there would also be one with $|R|=|B|$. That is, the 'special case' of (A) treated in [7] immediately entails the more general formulation in $(\mathrm{A})$.

\section{Balanced Lines and the GLBT}

We want to exhibit a more direct relation between (A) and (C). We will not do so with (C) itself, though, but replace it by the following assertion (E), which is known to be equivalent to $(\mathrm{C})$ by the Gale transform [9].

Fact $\mathbf{E} m \in \mathbf{N}$, even. Let $S$ be a set of $m$ points in 3-space, and let $\rho$ be a directed ray pointing at its apex $x$, such that $S \cup\{x\}$ is in general position, and $\rho$ is disjoint from $S$ and from all segments connecting points in $S$. An oriented triangle spanned by three points in $S$ is called a $j$-triangle of $S$, if there are exactly $j$ points of $S$ on its positive side. ${ }^{4}$ We say that $\rho$ enters a $j$-triangle $\Delta$ of $S$, if it intersects $\Delta$ from the positive side to the negative side of it (i.e., $x$ is on the negative side of $\Delta$ ). If $\rho$ crosses $\Delta$ from the negative to the positive side, then we say that $\rho$ leaves $\Delta$. Let $g_{j}(x, S)$ be the number of $j$-triangles entered by $\rho$ minus the number of $j$-triangles left by $\rho$.

$$
g_{m / 2-2}(x, S) \geq 0 .
$$

If $x$ is extreme in $S \cup\{x\}$ (but also in other situations), equality holds.

In fact, as already suggested by the above notation, it can be shown that $g_{j}(x, S)$ is a function of $x$ that is independent of the choice of the ray $\rho$ pointing at it. From this it immediately follows that if $x$ is extreme in $S \cup\{x\}$, then $g_{j}(x, S)=0$ for all $j$. Using the Gale transform (see [9]), one can show that if $x$ is not extreme in $S \cup\{x\}$, then there is a simple polytope $\mathcal{P}$ in $\mathbf{R}^{m-4}$ with at most $m$ facets, such that the so-called $g$-vector of $\mathcal{P}$ is exactly the vector $\left(g_{j}(x, S)\right)_{j=0}^{\lfloor(m-4) / 2\rfloor}$; see [9] for details (if $x$ is extreme, $\mathcal{P}$ is the empty polytope). The nonnegativity of this vector is the GLBT. ${ }^{5}$ We refer to [9] for the equivalence $(\mathrm{E} \Leftrightarrow \mathrm{C})$; see also [4].

We have prepared the ground for a proof of equivalence $(D \Leftrightarrow E)$. Assume the set-up of statement (D); recall that $Q$ has $2 n+1$ points. Put $Q_{0}=Q \backslash\left\{q_{0}\right\}$, and let $H(\tilde{q})$, for any $\tilde{q} \in \pi_{0}$, denote the number of halving triangles of $Q_{0} \cup\{\tilde{q}\}$ that are incident to $\tilde{q}$ and are crossed by $\pi_{0}$. We draw a line $\ell$ in $\pi_{0}$ passing through $q_{0}$, move a point $\tilde{q}$ along $\ell$ from infinity to $q_{0}$, and keep track of the changes in $H(\tilde{q})$ during this motion (see [2] for related results obtained this continuous motion paradigm, and [1, Chapter 3.6-3.8] for a thorough treatment of the combinatorial changes occurring in such a motion).

Initially, $\tilde{q}$ is an extreme point of $Q_{0} \cup\{\tilde{q}\}$ and so $H(\tilde{q})=\min \{k, 2 n-k\}$. As $\tilde{q}$ moves along $\ell, H(\tilde{q})$ changes only when $\tilde{q}$ becomes coplanar with three points $a, b, c \in Q_{0}$, so that the plane passing through these four points bounds two open halfspaces, one of which contains $n-1$ points of $Q_{0}$ and the other $n-2$. Three cases may arise, as illustrated in Figure 2.

(a) The four points $a, b, c, \tilde{q}$ are in convex position, say in this counterclockwise order, see Figure 2(a).

(b) The four points are not in convex position but $\tilde{q}$ is an extreme point of the quadruple, and, say, $c$ lies in the interior of $\tilde{q} a b$, see Figure 2(b).

\footnotetext{
${ }^{4}$ The orientation of the triangle declares one side of the plane it spans as the positive side. Obviously, the opposite orientation of a $j$-triangle is an $(m-3-j)$-triangle.

${ }^{5}$ And the characterization of all possible $g$-vectors is the $g$-Theorem.
} 

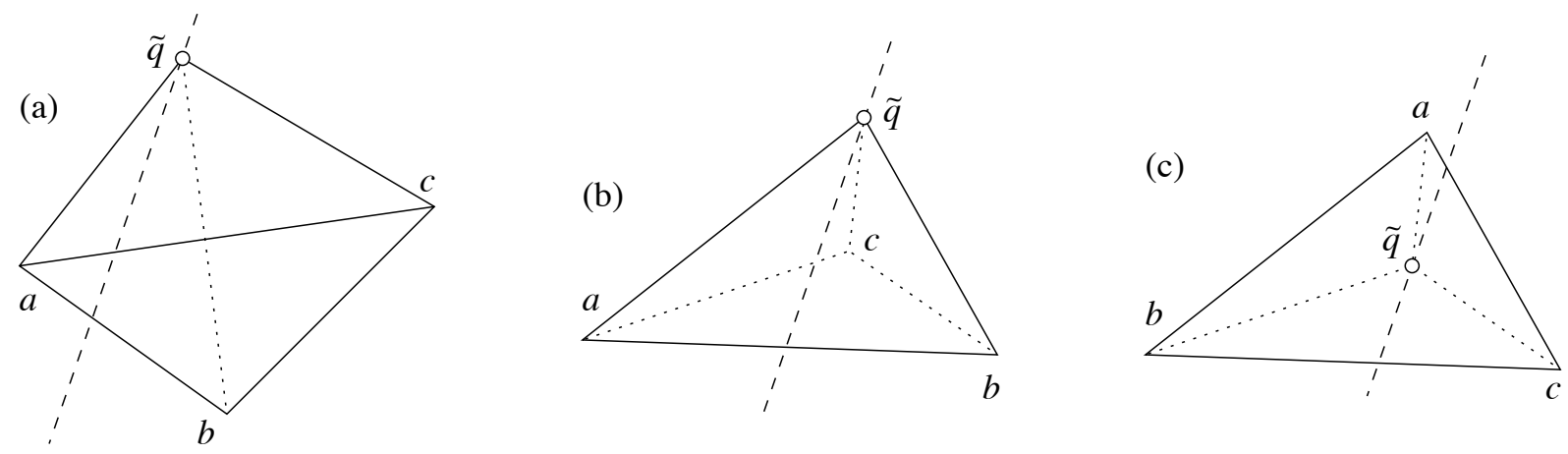

Figure 2: Three cases of coplanarity: (a) The four points are in convex position. (b) The four points are not in convex position but $\tilde{q}$ is an extreme point of the quadruple. (c) The four points are not in convex position and $\tilde{q}$ is the middle point.

(c) The four points are not in convex position and $\tilde{q}$ is the non-extreme ('middle') point, see Figure 2(c).

Each case is further divided into two subcases, depending on whether $\tilde{q}$ reaches the plane of $a b c$ from the side containing $n-2$ points of $Q_{0}$ (subcase (i)), or from the side containing $n-1$ points of $Q_{0}$ (subcase (ii)). Let $\delta$ denote the line of intersection of $\pi_{0}$ and the plane of $a b c$ (drawn as a dashed line in Figure 2).

In case (a.i), the triangles $a b c, \tilde{q} a c$ were halving triangles of $Q_{0} \cup\{\tilde{q}\}$ before $\tilde{q}$ reached the plane of $a b c$, and the triangles $\tilde{q} a b, \tilde{q} b c$ are halving triangles after $\tilde{q}$ leaves that plane. If $\delta$ does not cross the quadrangle $a b c \tilde{q}$ (at the time of coplanarity) then $\pi_{0}$ does not cross the triangle $\tilde{q} a c$ before $\tilde{q}$ reaches the plane of $a b c$, and does not cross $\tilde{q} a b$, qac after $\tilde{q}$ leaves that plane. Hence $H(\tilde{q})$ does not change in this case. On the other hand, if $\delta$ crosses $a b c \tilde{q}$ then $\pi_{0}$ crosses the triangle $\tilde{q} a c$ before $\tilde{q}$ reaches the plane of $a b c$, and crosses exactly one of the triangles $\tilde{q} a b, \tilde{q} a c$ afterwards. Hence $H(\tilde{q})$ does not change in this case either. Case (a.ii) is treated in a fully symmetric manner, and $H(\tilde{q})$ does not change in this subcase as well. In cases (b.i) and (b.ii) the local behavior at $\tilde{q}$ is the same as in the corresponding subcases (a.i) and (a.ii), so $H(\tilde{q})$ does not change in these cases either.

In case (c.i), the triangle $a b c$ was a halving triangle of $Q \cup\{\tilde{q}\}$ before $\tilde{q}$ reached the plane of $a b c$, and the triangles $\tilde{q} a b, \tilde{q} b c$ and $\tilde{q} a c$ are halving triangles after $\tilde{q}$ leaves that plane. The line $\delta$ always crosses exactly two of these three triangles, which means that $H(\tilde{q})$ increases by 2 in this subcase. By a symmetric reasoning, $H(\tilde{q})$ decreases by 2 in subcase (c.ii). In each of these subcases, abc spans, depending on its orientation, an $(n-2)$-triangle and an $(n-1)$-triangle of $Q_{0}$. In case (c.i), $\tilde{q}$ enters the $(n-2)$-triangle ${ }^{6}$ spanned by $a b c$, or more precisely, the ray on which $\tilde{q}$ moves to $q_{0}$ enters this $(n-2)$-triangle. In case (c.ii), $\tilde{q}$ leaves the $(n-2)$-triangle spanned by abc.

We have shown

$$
H\left(q_{0}\right)=\min \{k, 2 n-k\}+2 g_{n-2}\left(q_{0}, Q_{0}\right) .
$$

So $H\left(q_{0}\right) \geq \min \{k, 2 n-k\}$ iff $g_{n-2}\left(q_{0}, Q_{0}\right) \geq 0$. The latter is the assertion of (E). This completes the proof.

Remark 3.1. This implication does not hold if we consider the number of $j$-triangles of $Q$, for $j \leq n-2$, that are incident to $q_{0}$ and are crossed by $\pi_{0}$. In this case, $H(\tilde{q})$ changes by +2 when $\tilde{q}$ enters a $(j-1)$ triangle of $Q_{0}$ or when $\tilde{q}$ leaves a $j$-triangle of $Q_{0}$, and $H(\tilde{q})$ changes by -2 when $\tilde{q}$ leaves a $(j-1)$-triangle of $Q_{0}$ or when $\tilde{q}$ enters a $j$-triangle of $Q_{0}$. In this case we have

$$
H\left(q_{0}\right)=2 \min \{j+1, n-2-j, k, 2 n-k\}+2\left(g_{j}\left(q_{0}, Q_{0}\right)-g_{j-1}\left(q_{0}, Q_{0}\right)\right),
$$

which does not lead to the same implication as in the preceding proof.

\footnotetext{
${ }^{6}$ That is, $\tilde{q}$ approaches the plane of $a b c$ from the side that contains $n-2$ points of $Q_{0}$.
} 


\section{Discussion}

The purpose of this paper is to show the relation between the balanced lines problem (A) of [7], some problems involving halving triangles in 3-space, and the Generalized Lower Bound Theorem. This sheds some extra light on the result in [7] and explains the difficulty in obtaining a purely combinatorial proof of (A), as experienced in [7]. It highlights the additional merit of the proof of [7], in providing, implicitly, the first purely combinatorial proof of the special case of the Generalized Lower Bound Theorem described in (C). In doing so, we also obtained the property (D), which seems to be new. Several interesting challenges remain.

- Can one obtain a direct and simpler proof of the balanced lines result (A)?

- Can one obtain a purely combinatorial proof of the Generalized Lower Bound Theorem, beyond the special case established (indirectly) here?

\section{References}

[1] Artur Andrzejak, On k-Sets and their Generalizations, Ph.D. thesis (ETH No. 13441), ETH Zürich (2000).

[2] Artur Andrzejak, Boris Aronov, Sariel Har-Peled, Raimund Seidel, and Emo Welzl, Results on $k$-sets and $j$-facets via continuous motion, in "Proc 14th Ann. ACM Symp. on Comput. Geom." (1998), 192-199.

[3] Paul Erdős, László Lovász, A. Simmons, and Ernst G. Straus, Dissection graphs of planar point sets, in A Survey of Combinatorial Theory (J. N. Srivastava et al., Eds.), North Holland (1973), 139-149.

[4] Carl W.Lee, Winding numbers and the generalized lower-bound conjecture, DIMACS Series in Discrete Mathematics and Theoretical Computer Science 6 (1991), 209-219.

[5] László Lovász, On the number of halving lines, Ann. Universitatis Scientarium Budapest, Eötvös, Sectio Mathematica 14 (1971), 107-108.

[6] Peter McMullen, On simple polytopes, Inventiones Math. 113 (1993), 419-444.

[7] János Pach and Rom Pinchasi, On the number of balanced lines, Discrete Comput. Geom. 25 (2001), 611-628.

[8] Richard P. Stanley, The number of faces of simplicial polytopes and spheres, in "Discrete Geometry and Convexity", (J. E. Goodman, E. Lutwak, J. Malkevitch, and R. Pollack, Eds.), Annals New York Academy of Sciences 440 (1985), 212-223.

[9] Emo Welzl, Entering and leaving j-facets, Discrete Comput. Geom. 25 (2001), 351-364.

[10] Günter M. Ziegler, Lectures on Polytopes, Graduate Texts in Mathematics, Springer-Verlag (1995).

About Authors

Micha Sharir is at the School of Mathematical Sciences, Tel Aviv University, Tel Aviv 69978, Israel and at the Courant Institute of Mathematical Sciences New York University, New York, NY 10012, USA; sharir@math.tau.ac.il. Emo Welzl is at the Institute of Theoretical Computer Science, ETH Zürich, CH-8092 Zürich, Switzerland; emo@inf.ethz.ch. 


\section{Acknowledgments}

The authors thank Boris Aronov, János Pach, Rom Pinchasi, and Günter Ziegler for valuable discussions and remarks. A preliminary version of this paper has appeared in Proc. 17th Ann. ACM Symp. on Comput. Geom. (2001) 315-318. 